\title{
RDG System in Remote Areas with Utility or Local Feed Topology and Operation Strategy
}

\author{
Graciela M. Toccaceli ${ }^{1,2}$, Ricardo R. Peña ${ }^{2,3}$, Marcelo G. Cendoya ${ }^{1,2}$ and Pedro E. Battaiotto ${ }^{1}$ \\ 1. Facultad de Ingeniería, Universidad Nacional de La Plata, La Plata 1900, Argentina \\ 2. CONICET (Consejo Nacional de Investigaciones Cientificas y Técnicas), CABA C1033AAJ, Argentina \\ 3. Facultad de Ingeniería, Universidad Nacional de la Patagonia San Juan Bosco, Comodoro Rivadavia 9000, Argentina
}

Received: May 26, 2014 / Accepted: June 06, 2014 / Published: July 25, 2014.

\begin{abstract}
In remote regions with availability of wind energy, a RDG (renewable distributed generation) system is an advantageous alternative to increase the provision of electrical supply. Usually, these systems are structured on the basis of a connection to an existing weak grid. When the grid is out of service, the system may operate in islanding mode, if the RDG configuration allows it, continuing the provision of energy with standard voltage and frequency values. Facing the latter situation, a wind-diesel/gas generation system is proposed, with a conversion and control strategies based on a variable speed wind turbine employing a DFIG (doubly fed induction generator), a SC (ultracapacitor) storage system and a SG (synchronous generator) driven by a diesel/gas engine.
\end{abstract}

Key words: RDG, wind energy, diesel/gas, ultracapacitor, islanding operation.

\section{Introduction}

The supply of electricity in remote regions is usually done by a distribution AC (alternating current) grid, whose capacity in the medium and long term is insufficient to meet the growth demand (weak grid). In these cases, and where some form of renewable energy is available, distributed generation systems based on RDG (renewable distributed generation), constitute an advantageous alternative to locally increase the power capacity [1].

The RDG systems are normally designed to operate with grid connection, being the voltage and frequency values set by the grid. An alternative RDG topology can be achieved using a local synchronous generation complementary to that of the grid, which allows the system to operate in islanding mode, continuing the provision of energy when the grid goes down. In island operation, the RDG system must provide the standard

Corresponding author: Marcelo Gustavo Cendoya, M.Sc., professor, research fields: power electronics and renewable energy conversion systems. E-mail: cendoya@ing.unlp.edu.ar. voltage and frequency to the users by itself. In both operating modes is necessary the reduction of power fluctuations, in the grid or in the SG (synchronous generator), caused by the variable nature of the renewable resource and the load, in order to preserve the power quality (constant voltage and frequency).

Facing this kind of energy demand, electrical load and geographical location, this article proposes and analyzes a RDG system capable of operating in grid connection or islanding mode. The system structure, shown in Fig. 1, is based on the use of a variable speed wind turbine, an energy storage system with a SC (ultracapacitor) bank and a diesel/gas SG. The proposed RDG system and the results obtained in a first stage of its research and development are presented in the following order: Section 2 discusses the conversion strategy; Section 3 introduces the system modeling; Section 4 shows the evaluation by simulation of the system operating strategy; and finally, in Section 5, remarks and conclusions are summarized. 


\section{Conversion Strategy}

A conversion strategy consists mainly of two parts:

- The structure of the system;

- The operation and control of the system.

\subsection{System Structure}

The proposed RDG system is presented in Fig. 1, it consists of three modules linked through a local AC bus: wind turbine, storage system and diesel/gas SG. The AC load is also shown.

A variable pitch three-bladed horizontal axis wind turbine performs the wind-mechanical conversion. A three-phase DFIG (doubly fed induction generator) with a static Kramer drive, performs the mechanical-electrical conversion. It is possible to vary the wind turbine power contribution by acting on the firing angle $\alpha$ of the controlled rectifier. In this conversion topology, the power managed by the converters is a fraction of the total generated power, resulting in an inexpensive and simple implementation. It is possible to use low-cost wind turbines available from the repowering market.

A storage system based on a SC bank [2-7], is connected to the AC bus through a three-phase voltage inverter, with series inductors in each phase. A closed loop control the power handled by the storage system, imposing the DC (direct current)-side inverter current. Power flow may be bidirectional. The SC bank is linked to the inverter via a bi-directional DC-DC boost converter that maintains a constant DC link voltage.

The SG, driven by a diesel or gas turbine engine, powered the local AC bus when the grid is disconnected. The conventional voltage and frequency control systems are associated to it, acting on the field and rotation speed, respectively.

\subsection{Operation Mode and System Control}

\subsubsection{Wind Turbine Control}

The temporal evolution of the turbine rotational speed $\omega$, is defined by:

$$
T_{T}-T_{G}=J \mathrm{~d} \omega / \mathrm{d} t+B \omega
$$

where, $T_{T}, T_{G}$ : turbine and generator torques. $J, B$ : inertia moment and friction coefficient of the ensemble.

Neglecting friction, the operating point of the turbine at steady state $(\omega=$ constant $)$ is defined by:

$$
T_{T}=T_{G}
$$

To work at a desired point, the generator should impose $T_{G}$, which is achieved by a control loop acting on $\alpha$, setting the DFIG rotor current. Saturation is included to avoid exceeding the nominal rotor current. $T_{G}$ is measured indirectly, by sensing only electrical variables. In asynchronous machines, if the iron and copper losses in stator are neglected, it holds that:

$$
P_{S}=P_{G a p}=T_{G} \omega_{s} \Rightarrow T_{G}=\frac{v_{a} i_{a}+v_{b} i_{b}+v_{c} i_{c}}{\omega_{s}}
$$

with, $P_{S}$ : stator power; $P_{\text {Gap }}$ : gap power; $v_{a}, v_{b}, v_{c}$ and $i_{a}$, $i_{b}, i_{c}$ : stator voltages and currents; $\omega_{s}=2 \pi f / p$ : synchronous speed; $f$ : grid frequency and $p$ : pole pairs.

The reference $T_{G}{ }^{*}$ for the torque control loop is determined from desired power of the wind turbine $P_{g}{ }^{*}$, and, the generator rotation speed $\omega_{g}$, as:

$$
T_{G}^{*}=P_{g}^{*} / \omega_{g}
$$

$P_{g}{ }^{*}$ is different for each operation mode of the RDG system, which is determined by the position of switches $\mathrm{K} 1$ and $\mathrm{K} 2$.

Different aspects of the turbine control are detailed below:

(1) Operation with grid connection

In this operation mode, the switches $\mathrm{K} 1$ and $\mathrm{K} 2$ are in the "G" position and the turbine delivers the maximum possible power, taking full advantage of the available wind resource. The surplus power not consumed by the AC load, is injected into the grid. The turbine maximum power is reached when the power coefficient $C_{p}$ attain its optimum value. To obtain this, the turbine must rotate at a particular speed for each wind speed, which is determined by the optimum tip speed ratio $\lambda_{0}$. The points that correspond to maximum power describe a cubic parabola in the $P-\omega$ plane, given by: 


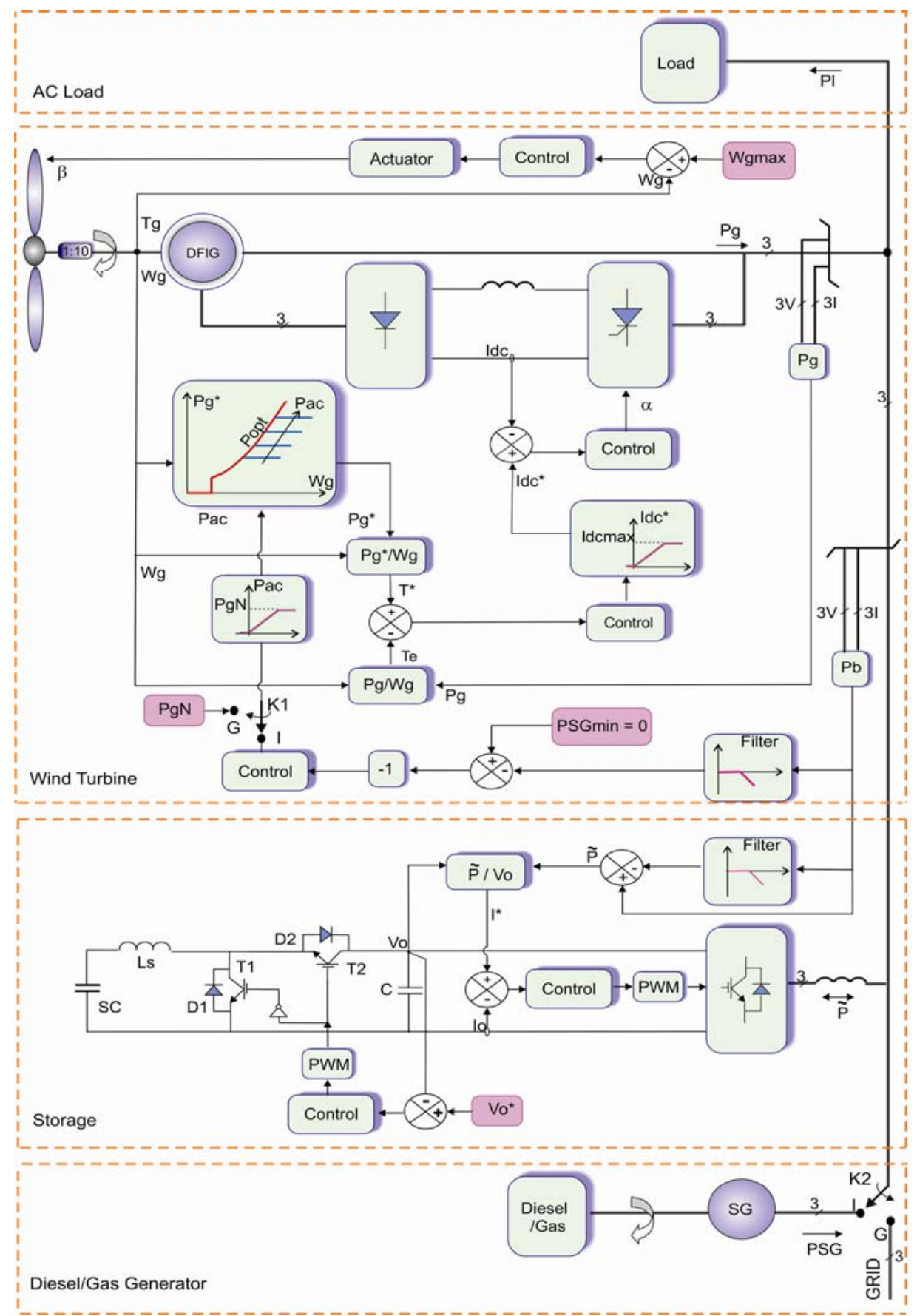

Fig. 1 RDG system structure.

$$
P_{T \max }=C_{P \max }\left(\lambda_{0}\right) P_{w}=K_{p} \omega^{3}
$$

$$
P_{g}^{*}=P_{\operatorname{Tmax}}
$$

where, $P_{T}$ : turbine power; $C_{p}(\lambda)$ : power coefficient; $\lambda=$ $r \omega / v ; r$ : blade radius; $\omega$ : turbine rotational speed; $P_{W}=$ $0.5 \rho A v^{3}$ : wind power; $\rho$ : air density; $A=\pi r^{2}$ : capture area; $v$ : wind speed; and $K_{p}$ : constructive constant.

Therefore, the reference power of the generator is:

\section{(2) Islanding operation}

The switches K1 and K2 are in the position "I" in this operation mode, and the wind turbine is intended to provide the power consumed by the AC load, $P_{l}$. The SG will provide power only when the wind generation 
is insufficient. In this way, fuel consumption and environmental pollution are minimized.

The turbine control loop regulates the AC bus power $P_{b}$, so that no power is requested from the SG. It follows that, if the wind resource is sufficient, the turbine will provide:

$$
P_{S G}=0 \Rightarrow P_{g}=P_{l}
$$

The measurement of $P_{S G}$ incorporates a low-pass filter, thus the control loop regulates only its average value.

Noting the typical wind energy spectrum [8] from Fig. 2, it is convenient to choose a filter cut-off frequency of about one cycle/h $(0.3 \mathrm{mHz})$.

Furthermore, the control loop has saturation, $P_{g N}$, to avoid exceeding the generator rated power.

(3) Maximum speed limitation

A control loop acts on the pitch angle of the turbine blades, $\beta$, to prevent overcome of the rotation speed limit that could damage the mechanical integrity of the system. If the rotational speed is below the limit value, the blade pitch is maintained at $\beta=0^{\circ}$. When the speed tries to exceed the limit value, $\beta$ is increased to maintain a constant $\omega_{g}$.

(4) Wind turbine operation region

The proposed control strategies for the wind turbine, determine an operation region in the torque-speed plane of Fig. 3. It is limited by the quadratic parabola of optimal pair $T_{o p t}$; the hyperbola corresponding to the constant power load $P_{l}$, and the vertical line corresponding to the maximum permissible speed. It thus excludes all operating points (transient or steady), that may cause eventual blockage of the turbine to operate in a non-controllable area.

$T_{T}=f(\omega, v)$ and $T_{G}=f\left(\omega, P_{g}^{*}\right)$ are shown in Fig. 3. In grid connection mode, the operating point (intersection of $T_{T}$ with $T_{G}$ ), will fall on the $T_{\text {opt }}$ parabola, taking full advantage of the wind resource $\left(P_{\operatorname{gmax}}\right)$.

In islanding mode operation, and with sufficient wind resource, the operating point will be located on the hyperbola corresponding to the load power $P_{l}$ and

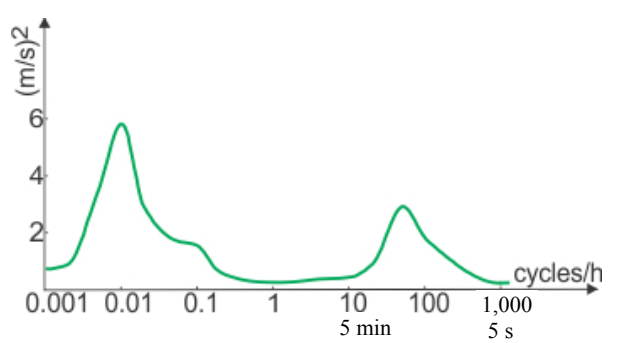

Fig. 2 Typical wind energy spectrum.

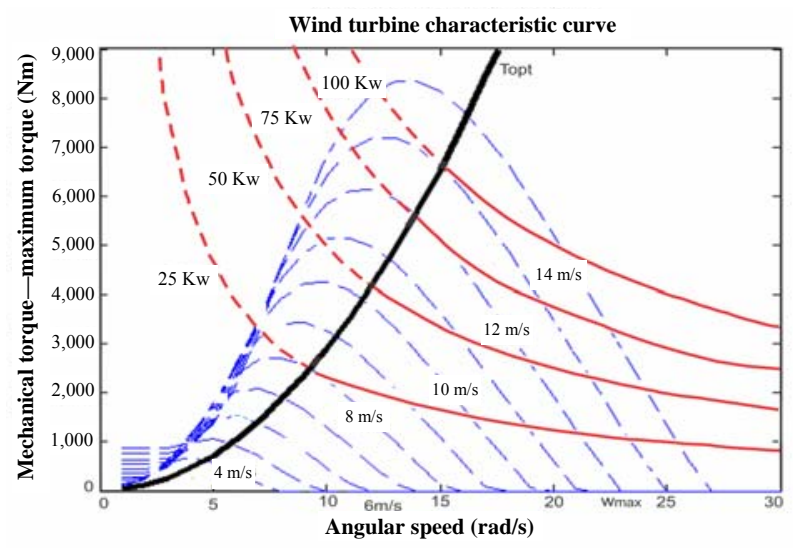

Fig. 3 Curves of the turbine and the generator.

the SG does not supply power. If the wind resource is insufficient, the operating point lies on the parabola of $T_{\text {opt }}$, which corresponds to $\left(P_{\text {gmax }}\right)$ and the SG supplies the power difference $\left(P_{l}-P_{\operatorname{gmax}}\right)$.

\subsubsection{Storage System Control}

The storage system is operated to reduce disturbances in the power transmitted to the grid or the SG, by rapid changes in the wind speed due to gusts, or abrupt load variations. By subtracting the slowly varying component $\bar{P}$ (obtained by low-pass filtering) to the total power $P$, the fluctuating component of power is determined. This component is the power that the storage system must store/deliver, namely:

$$
P_{S C}=P-\bar{P}
$$

Noting the spectrum of the wind energy, in Fig. 2, the turbulent zone is located over the frequency of 10 cycles/hour, making it suitable a filter with a cut-off frequency of $3 \mathrm{mHz}$.

To make that the power of the storage system corresponds to the given in Eq. (8), a current control loop is used in the DC side of the inverter, acting on its 
PWM modulation index. The reference for this loop is calculated as follows:

$$
I^{*}=P_{S C} / V_{0}
$$

On the other hand, a control loop set the duty cycle (D) of the bidirectional DC-DC converter to maintain a constant DC-link voltage. This allows a proper operation of the inverter for any SC bank voltage, which varies according to the stored energy level.

\section{System Modeling}

The models for each component of the RDG system are presented below.

\subsection{Wind Turbine}

\subsubsection{Mechanical Subsystem}

Eq. (1) is used with $T_{T}$ calculated by:

$$
P_{T}=T_{T} \omega \Rightarrow T_{T}=0.5 \rho A r v^{2} C_{p}(\lambda, \beta) / \lambda
$$

where, the power coefficient $C_{P}(\lambda, \beta)$ taken from Ref. [9] is:

$$
C_{p}(\lambda, \beta)=c_{1}\left(c_{2} / \lambda_{i}-c_{3} \beta-c_{4}\right) e^{-\frac{c_{5}}{\lambda_{i}}}+c_{6} \lambda
$$

with, $\lambda_{i}^{-1}=(\lambda+0.08 \beta)^{-1}-0.035\left(\beta^{3}+1\right)^{-1}$, and $c_{1}=$ $0.5176, c_{2}=116, c_{3}=0.4, c_{4}=5, c_{5}=21, c_{6}=0.0068$, giving $C_{p \max }=0.48$ for $\lambda_{0}=8.1$ and $\beta=0$.

\subsubsection{DFIG-Kramer Drive}

For the DFIG, a $d q$ classic model is used [10], which is available in the library of the simulation software employed. As the controlled and uncontrolled rectifiers of the static Kramer drive operate at low frequencies (grid and rotor frequencies), they are represented by electrical circuits and real electronic components, available in the library mentioned above.

\subsection{Storage System}

\subsubsection{SC bank-DC-DC Converter}

The SC bank is represented by an equivalent capacity $C_{S C}$ and a parasitic series resistance $R_{S C}$. Thus the $\mathrm{SC}$ bank terminal voltage is:

$$
v_{s c}=R_{S C} i_{S C}+\frac{1}{C_{S C}} \int i_{S C} \mathrm{~d} t
$$

As the bidirectional DC-DC converter operates at a high switching frequency, a simplified model is used, in order to reduce the simulation time. This model is valid in continuous conduction mode, and takes into account the voltage and current values averaged over a switching cycle. At the SC bank side, the converter is modeled with an ideal voltage source $D V_{0}$. Therefore, the charge/discharge of the SC bank is governed by:

$$
D V_{0}=L \frac{\mathrm{d}}{\mathrm{d} t} i_{S C}+R_{S C} i_{S C}+\frac{1}{C_{S C}} \int i_{S C} \mathrm{~d} t
$$

In the DC link side, the converter is represented by a controlled current source. If a perfect lossless converter it is assumed, the current can be calculated with:

$$
i_{\mathrm{o}}=D i_{S C}
$$

\subsubsection{Inverter}

As the PWM inverter operates at a high switching frequency, then, following the same approach used to model the DC-DC converter, a functional equivalent macro model is used. The grid side is represented by three controlled current sources:

$$
\left\{\begin{array}{l}
i_{a}=\hat{I} \sin (\omega t) \\
i_{b}=\hat{I} \sin \left(\omega t-120^{\circ}\right), \hat{I}=\frac{2}{3} \frac{\eta_{i n v} P_{S C}}{\widehat{V}_{A C}} \\
i_{c}=\hat{I} \sin \left(\omega t+120^{\circ}\right)
\end{array}\right.
$$

where, $\eta_{i n v}$ : inverter efficiency, $\widehat{V}_{A C}$ : grid peak voltage and $\omega=2 \pi f$ : grid angular frequency.

In the DC-link side, the inverter is represented by a controlled current source, whose value is given in Eq. (9).

\subsection{Diesel/Gas Synchronous Generator}

It is assumed that the voltage and frequency control loops (acting on the field current and rotation speed, respectively) have zero error, and therefore the SG is modeled as three ideal voltage sources with series impedances, generating: 


$$
\left\{\begin{array}{l}
v_{a}=\hat{V}_{A C} \sin (\omega t) \\
v_{b}=\hat{V}_{A C} \sin \left(\omega t-120^{\circ}\right) \\
v_{c}=\hat{V}_{A C} \sin \left(\omega t+120^{\circ}\right)
\end{array}\right.
$$

\section{Conversion Strategy Evaluation}

The proposed RDG system was simulated in the MATLAB $® /$ Simulink environment, employing the models presented in Section 3. The values of the parameters used for these models are given below:

- Wind turbine

$P_{N}=100 \mathrm{KW}$ for $v=14 \mathrm{~m} / \mathrm{s}, r=7 \mathrm{~m}, \rho=1.2242$ $\mathrm{kg} / \mathrm{m}^{3}$.

\section{- DFIG}

$P_{N}=110 \mathrm{KW}$ and $\omega_{N}=1,487 \mathrm{rpm}$. Stator:

$V_{N}=400 \mathrm{~V}$ and $f_{N}=50 \mathrm{~Hz}, P=4$.

- SC bank

$C_{S C}=27 \mathrm{~F}, R_{S C}=0.06 \Omega, V_{S C}=135-270 \mathrm{~V}(100$ cells in series).

Four different test situations are evaluated, the first two being for the RDG system connected to the grid and the remaining two for islanding mode. The results are presented in the torque-speed plane of the turbine, with indicative trajectories of the power variation in the different parts of the system. The instantaneous electrical powers associated with each component of the system, corresponding to these trajectories, are also depicted as power versus time waveforms.

\subsection{Case 1: RDG in Grid Connection}

When the system is connected to the grid, switches in position "G" (Fig. 1), the wind turbine is controlled to operate on the optimum torque curve $\left(T_{\text {opt }}\right)$ for different wind speeds (Fig. 4), producing a maximum wind-electric energy conversion. SC storage system is operated to filter rapid fluctuations in $\mathrm{AC}$ bus power, preventing them to reach the "weak grid", disturbing its power quality.

Two different situations, $1 \mathrm{a}$ and $1 \mathrm{~b}$, which correspond to sinusoidal variations of wind power $\left(P_{g}\right)$ or load $\left(P_{l}\right)$ power are considered. The evolution of grid power $P_{\text {grid }}$ and storage system power $P_{S C}$, are then analyzed. As presented in Fig. 5, variations in $P_{g}$ (case 1a) or $P_{l}$ (case 1b) between $25 \mathrm{KW}$ and $75 \mathrm{KW}$ are proposed. Two frequency values were chosen for these power variations, which correspond to the peaks of the wind energy spectrum that is shown in Fig. 2.

\subsubsection{Case 1a: Variable Wind and Constant Load}

To make interpretation of the results easier, a load power $P_{l}=50 \mathrm{KW}$ is considered, which corresponds to the turbine steady-state operating point "A", as shown

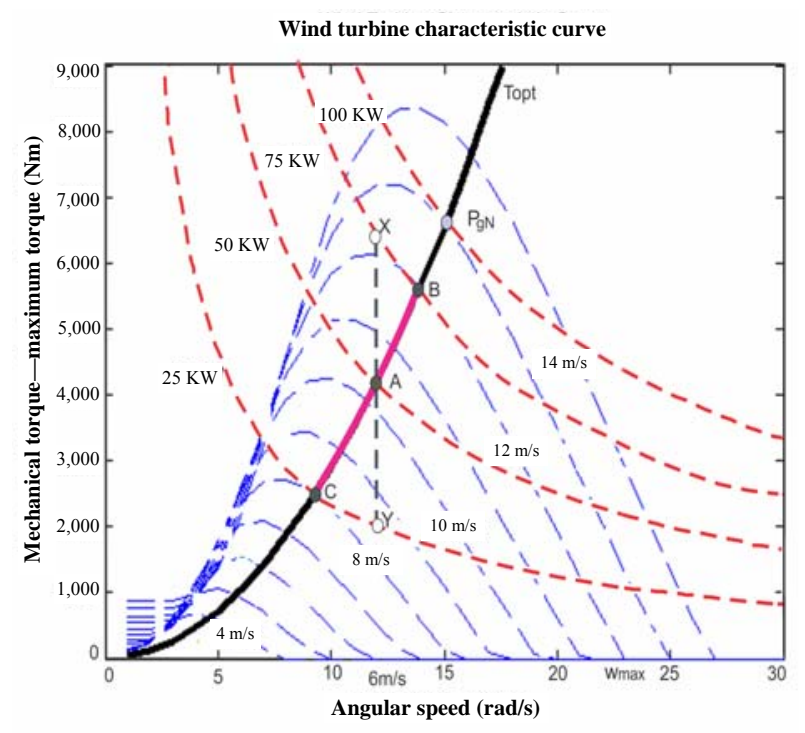

Fig. 4 Operating points in case $1 \mathrm{a}$ and case $1 \mathrm{~b}$.
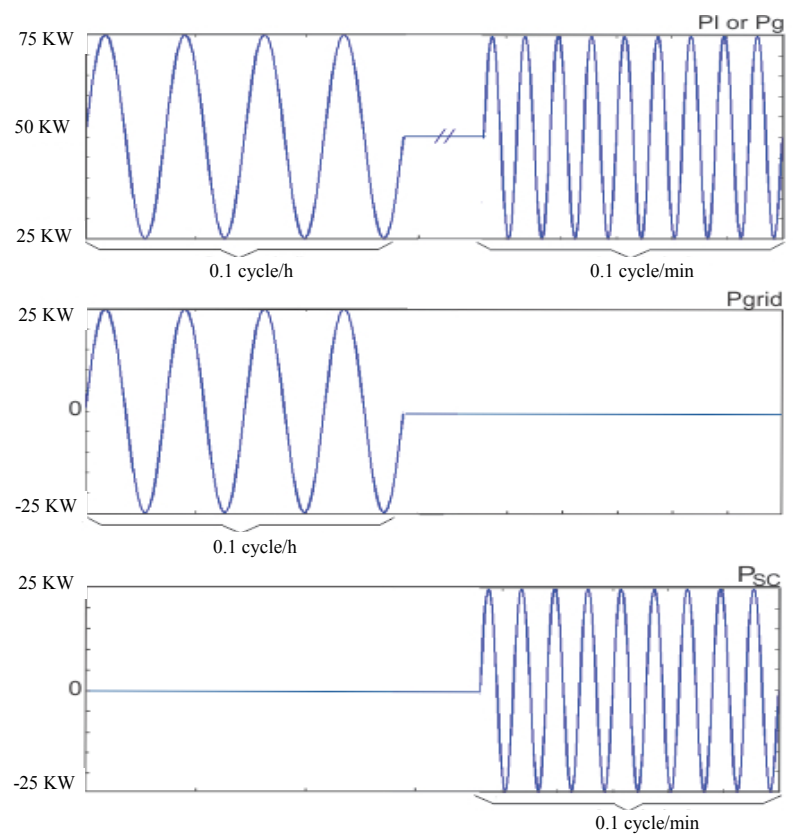

Fig. 5 Electrical powers in case $1 \mathrm{a}$ and case $1 \mathrm{~b}$. 
in Fig. 4. For frequency fluctuations both of $0.1 \mathrm{cycle} / \mathrm{h}$ and $1 \mathrm{cycle} / \mathrm{min}$, the wind turbine operating points are located on the "C-A-B" segment, with wind speeds between $8 \mathrm{~m} / \mathrm{s}$ and $12 \mathrm{~m} / \mathrm{s}$. Filtering, due to the inertia of the mechanical structure, is negligible at these frequencies.

The wind power $P_{g}$, injected into the AC bus of the RDG system, is shown in Fig. 5. The value of $50 \mathrm{KW}$ is taken by the load, and sinusoidal fluctuations are supplied by the grid or the storage system, depending on the frequency value. The storage system control loop only responds to frequencies corresponding to the turbulence spectrum $(f>10$ cycles/h). It is observed that the turbulence does not disturb the grid and its energy is harnessed by the storage system to power the load when wind speed is less than $11 \mathrm{~m} / \mathrm{s}$.

\subsubsection{Case 1b: Constant Wind and Variable Load}

For simplicity in the interpretation of the results, in this case it is considered a wind power of $P_{g}=50 \mathrm{KW}$, that corresponds to a constant wind speed of $11 \mathrm{~m} / \mathrm{s}$. Under these conditions, the turbine will remain at point " $A$ " while not occur a change in wind speed, as seen in Fig. 4. The fluctuating power $P_{l}$, indicated in Fig. 5, is taken from the AC bus of the RDG system. As a constant power of $50 \mathrm{KW}$ is provided by the wind turbine, the sinusoidal fluctuations are supplied by the grid or by the storage system, according to its frequency value.

The storage system control loop only responds to frequencies corresponding to the spectrum of turbulence. It is noted that the fast disturbances caused by the load, in that frequency range, do not disturb the grid and its energy is harnessed by the storage system to power the load when it requires a greater than its average power of $50 \mathrm{KW}$, delivered by the wind turbine.

In Fig. 4, the points on the "Y-A-X" segment represent the difference between load power and wind power, which reaches its maximum value of in $25 \mathrm{KW}$ at points " $X$ " and "Y".

\subsection{Case 2: RDG in Islanding Mode}

In this mode, the RDG system is isolated from the grid and connected to the local SG, switches in "I" position, see Fig. 1. Since the SG cannot receive power, the wind turbine is operated to provide $P_{\text {gmax }}$ for each value of wind, as in the case of grid connection. This holds until the SG delivers null power $\left(P_{S G}=0\right)$. From this condition, the wind turbine operates maintaining $P_{S G}=0$ condition in the $\mathrm{SG}$ and delivering full power to the load. While the turbine operates as a regulator of $P_{S G}=0$, all power fluctuations by wind variation, independently of the frequency, are rejected by the torque control loop, and will not be injected into the AC bus of the RDG. The storage system is operated in the same way as in grid connection, to filter out rapid fluctuations in AC bus power, avoiding arrival to SG.

Two different situations are then analyzed, showing the evolution of the wind turbine $\left(P_{g}\right)$, the SG $\left(P_{S G}\right)$, and the storage system $\left(P_{S C}\right)$ powers against variations of wind or load power. For analysis and simulation, variations in wind speed (case 2a) or load power (case $2 b$ ) are proposed, for two different frequencies in correspondence with the peaks in the spectrum presented in Fig. 2.

4.2.1. Case 2a: Variable Wind and Constant Load

The load power considered is $P_{l}=50 \mathrm{KW}$, which corresponds to the maximum wind turbine power $\left(P_{\text {gmax }}\right)$ for a wind speed close to $10 \mathrm{~m} / \mathrm{s}$, as shown in Fig. 6.

For higher wind speed, the value of $P_{g}$ is maintained in $50 \mathrm{KW}$, and wind turbine operating points are located in the "C-A-B" segment of the constant power hyperbola, up to $13 \mathrm{~m} / \mathrm{s}$. In this range of operation, the powers of SG and SC are maintained at "zero", since there is no change in $\mathrm{AC}$ bus power. The power control loop of the wind turbine rejects disturbances due to wind variations.

For wind speed lower than $10 \mathrm{~m} / \mathrm{s}$, such as the of 9 $\mathrm{m} / \mathrm{s}$ value used for the simulation, $P_{g}$ is less than 50 $\mathrm{KW}$. The difference between $P_{l}$ and $P_{g}$ is provided by SG $\left(P_{S G}\right)$ and SC $\left(P_{S C}\right)$ in a similar way as case 1 , with grid connection. 


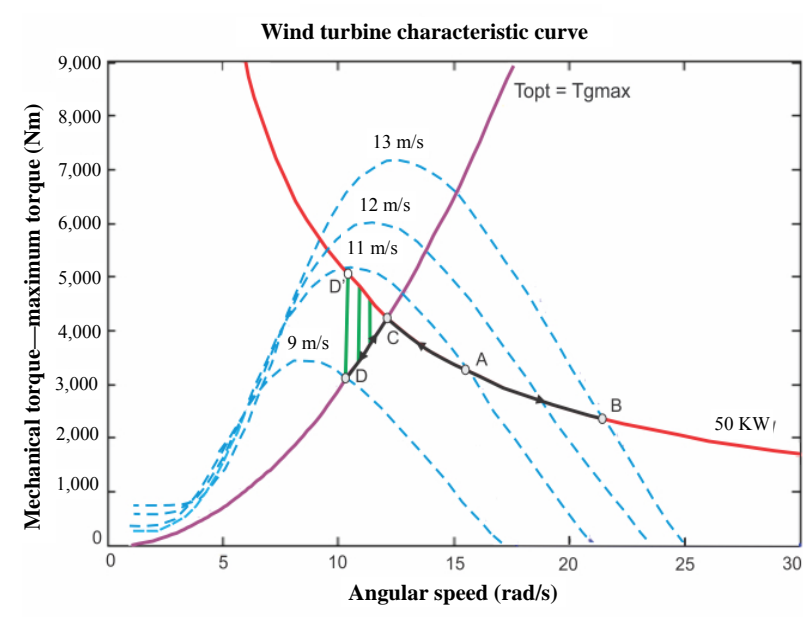

Fig. 6 Operating points in case 2a.

\subsubsection{Case 2b: Constant Wind and Variable Load}

A constant wind speed of $12 \mathrm{~m} / \mathrm{s}$ is considered, which allows a $P_{\text {gmax }}=75 \mathrm{KW}$, point "B" in Fig. 7. The load power $P_{l}$ varies sinusoidally between $25 \mathrm{KW}$ and $75 \mathrm{KW}$, with a frequency of $0.1 \mathrm{cycle} / \mathrm{h}$ or $1 \mathrm{cycle} / \mathrm{min}$, as in the case 1, and is presented in Fig. 8. The average load power of $50 \mathrm{KW}$ and its slow fluctuations are entirely provided by the wind turbine, shifting its operating point on the "C-A-B" segment in Fig. 7. Rapid load fluctuations are provided by the SC, and points on the "Y-A-X" segment represent the difference between the average load power provided by the wind turbine and the total load power, see Fig. 7. It reaches its maximum value of $25 \mathrm{KW}$ in " $\mathrm{X}$ " and " $\mathrm{Y}$ " points. For any load power level less than $75 \mathrm{KW}$, which is the maximum $P_{g}$, SG remains at $P_{S G}=0$.

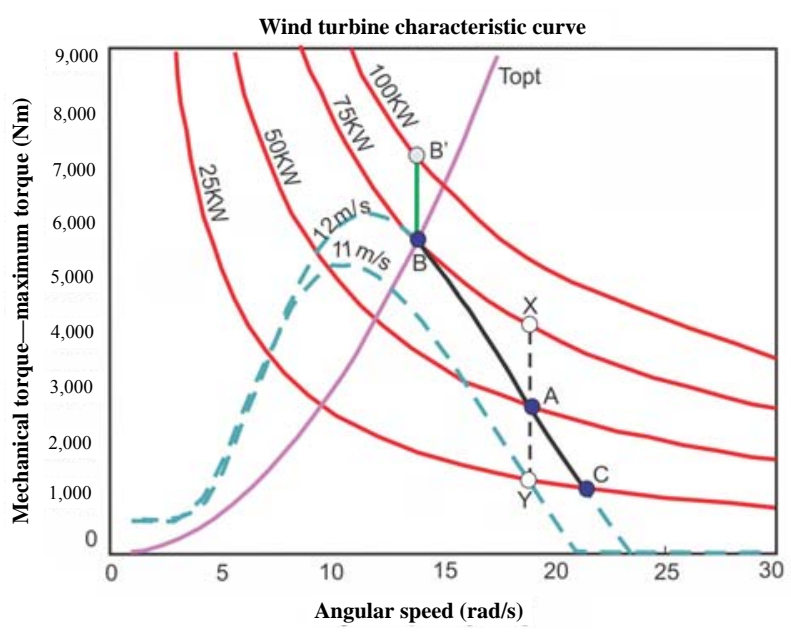

Fig. 7 Operating points in case $2 b$.

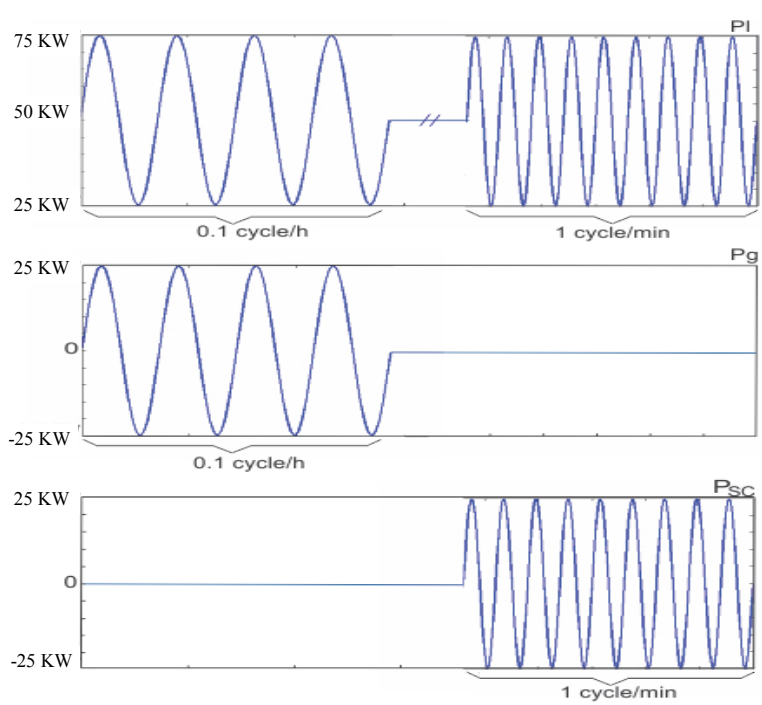

Fig. 8 Electrical powers in case $2 b$.

When $P_{l}$ exceeds $75 \mathrm{KW}$, the wind turbine is kept in the point of operation "B", delivering up to $75 \mathrm{KW}$, while the difference is provided by the $\mathrm{SG}\left(P_{S G}\right)$ and the storage system $\left(P_{S C}\right)$.

\section{Conclusions}

The obtained results integrally validate the proposal. Convergence to the expected steady state values was found. It is noted that both the optimum tuning of the controllers, as well as the system stability analysis, will be addressed in a future work.

The operation and control strategy allows maintaining an adequate power quality, even with high wind-energy penetration.

As the system components are of a standard technology and of relatively low-cost, the proposed RDG system is affordable and applicable in multiple contexts with weak grids.

Due to its islanding operation capability, the system is particularly useful in areas with frequent interruptions in the electrical service.

\section{Acknowledgments}

This work has been performed through the support of CONICET, UNLP and UNPSJB.

\section{References}

[1] Keyhani, A., Marwali, M. N., and Dai, M. 2010. 
Integration of Green and Renewable Energy in Electric Power Systems. USA: Wiley.

[2] Aghatehrani, R., Kavasseri, R., and Thapa, R. C. 2010. "Power Smoothing of the DFIG Wind Turbine Using a Small Energy Storage Device." Presented at the IEEE PES General Meeting, Minnesota, USA.

[3] Luu, T., and Nasiri, A. 2010. "Power Smoothing of Doubly Fed Induction Generator for Wind Turbine Using Ultracapacitors." In Proceedings of IECON 2010, 3293-98.

[4] Onar, O. C., Uzunoglu, M., and Alam, M. S. 2006. "Dynamic Modeling, Design and Simulation of a Wind/Fuel Cell/Ultra-Capacitor-based Hybrid Power Generation System.” JPS 161: 707-22.

[5] Rashed, M., Ghanem, A. E, H., and Sayes, A. E. 2009. "Control Strategy for an Isolated DFIG Based Micro-grid with Integrated Super-capacitors." OJEEE 1 (2): 81-8.

[6] Tankari, M. A., Camara, M. B., Dakyo, B., and Nichita, C. 2011. "Ultracapacitors and Batteries Integration for Power Fluctuations Mitigation in Wind-PVDiesel Hybrid System." IJRER 1 (2): 86-95.

[7] Zhou, T., and François, B. 2011. "Energy Management and Power Control of a Hybrid Active Wind Generator for Distributed Power Generation and Grid Integration.” IEEE TIE 58 (1): 95-104.

[8] Bianchi, F. D., De Battista, H., and Mantz, R. J. 2006. Wind Turbine Control Systems. London: Springer.

[9] Heier, S. 1998. Grid Integration of Wind Energy Conversion Systems. NY: John Wiley \& Sons Ltd..

[10] Krause, P. C., Wasynczuk, O., Sudhoff, S. D. 2002. Analysis of Electric Machinery and Drive Systems. USA: Wiley IEEE Press. 